\title{
The First Case of Chronic Otitis Media due to Kerstersia gyiorum in Korea
}

\author{
Jin-Hong Kim, M.D., Eunyoung Lee, B.S., and Yangsoon Lee 마, M.D., Ph.D. \\ Department of Laboratory Medicine, Hanyang University Seoul Hospital, Hanyang University College of Medicine, Seoul, Korea
}

\section{Dear Editor,}

Kerstersia gyiorum is a pathogen that is infrequently reported in humans [1]. This bacterium, first classified in 2003, is a gramnegative coccobacillus belonging to the family Alcaligenaceae, which is isolated from water, soil, animal, and human specimens [2, 3]. Although some cases of $K$. gyiorum infection have been reported worldwide [4-8], none to date have been reported in Korea. We report the first case of chronic otitis media (COM) due to $K$. gyiorum infection in Korea.

A 51-year-old female patient visited the otolaryngology department of Hanyang University Seoul Hospital with otorrhea in the left ear, which had started several weeks earlier. At the age of 25 and 28 years, she underwent canal wall-down mastoidectomies type III tympanoplasty of the right and left ears, respectively. On the day of admission, ear endoscopy revealed a purulent discharge and crust in the left external ear canal; therefore, the patient received crust removal and cefpodoxime treatment.

A swab specimen was taken from the patient's ear discharge and analyzed by bacterial culture and Gram staining. Gram staining revealed gram-negative coccobacilli in singles, pairs, and short chains (Fig. 1A). The specimen was inoculated on $5 \%$ sheep blood agar and MacConkey agar. After 24 hours, colonies of the organism grew on both sheep blood agar and MacConkey agar at $35^{\circ} \mathrm{C}$ under $5 \% \mathrm{CO}_{2}$ (Fig. $1 \mathrm{~B}$ and $1 \mathrm{C}$ ). The colonies were gray and displayed spreading edges; colony isolates were oxi- dase negative and catalase positive. Isolates were examined using the MicroScan Walkaway (Beckman Coulter, Brea, CA, USA) and VITEK 2 (bioMérieux, Marcy-l'Etoile, France) automated bacterial identification systems; however, both analyses failed to identify the bacterial species. The species was identified as $K$. gyiorum by matrix-assisted laser desorption/ionization-time of flight mass spectrometry (MALDI-TOF MS) with a MALDI Biotyper using MALDI-Biotyper software (version 2.3, Bruker Daltonics, Bremen, Germany). 16S ribosomal RNA gene sequencing was performed using primers 27F (5'-AGAGTTTGATCCTGGCTCAG-3') and 1492R (5'-GGTTACCTTGTTACGACTT-3') as described previously [1]. We obtained a consensus sequence of 1,394 bp, which was 99.9\% identical to K. gyiorum LMG 5906 (GenBank accession number AY131213). The next-nearest match was K. similis LMG 5890 (GenBank accession number AY131212), with $99.4 \%$ homology. In addition, gyrB gene sequencing was performed as described previously [4]. The gyrB gene sequence was $98.6 \%$ and $96.9 \%$ identical to $K$. gyiorum LMG 5906 (GenBank accession number HE585644) and K. similis LMG 5890 (GenBank accession number HE585647), respectively.

The results of the antimicrobial susceptibility test performed with the MicroScan Walkaway system were assessed according to the Clinical and Laboratory Standards Institute (CLSI) minimum inhibitory concentration (MIC) standards for other non-
Received: December 5, 2017

Revision received: March 13, 2018

Accepted: June 19, 2018

\section{Corresponding author: Yangsoon Lee}

(iD https://orcid.org/0000-0003-3821-3741

Department of Laboratory Medicine, Hanyang University Seoul Hospital, Hanyang University College of Medicine, 222-1 Wangsimni-ro, Seongdonggu, Seoul 04763, Korea

Tel: +82-2-2290-9655, Fax: +82-2-2290-9193

E-mail: yangsoon@hanyang.ac.kr

\section{(C) Korean Society for Laboratory Medicine}

This is an Open Access article distributed under the terms of the Creative Commons Attribution Non-Commercial License (http://creativecommons.org/licenses/by-nc/4.0) which permits unrestricted non-commercial use, distribution, and reproduction in any medium, provided the original work is properly cited. 

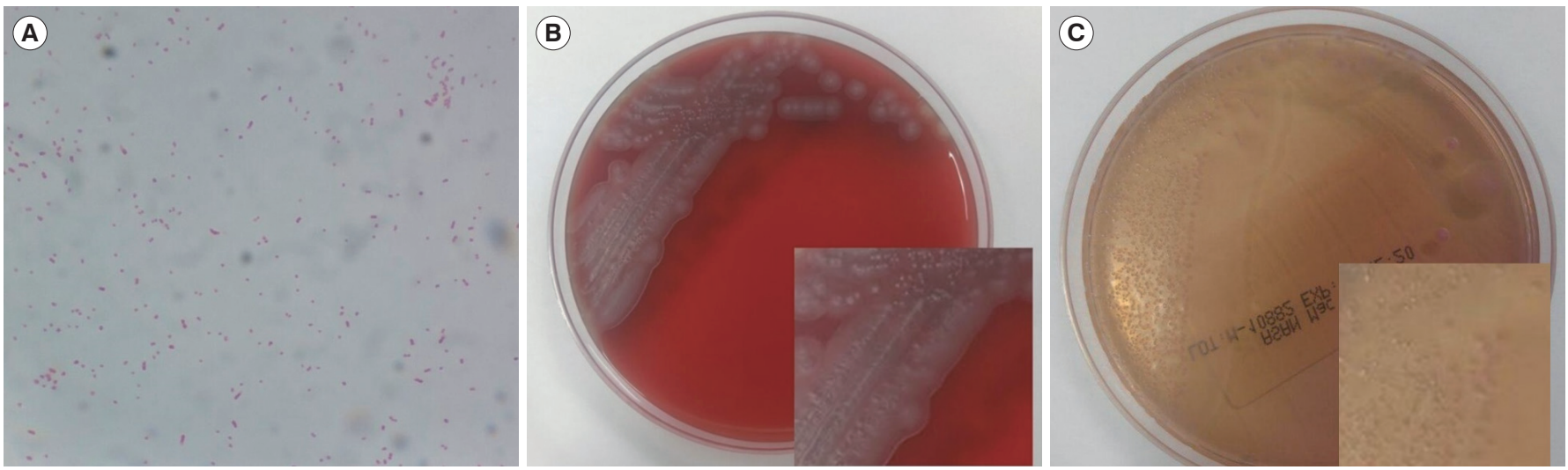

Fig. 1. Microscopic and colony morphology of Kerstersia gyiorum. (A) Gram-negative coccobacilli on a Gram-stained smear ( $\times 1,000)$. (B) Gray colonies with spreading edges on $5 \%$ sheep blood agar after 24-hour incubation. (C) K. gyiorum obtained from the patient's specimen on MacConkey agar.

Table 1. Summary of Kerstersia gyiorum cases related to chronic otitis media

\begin{tabular}{|c|c|c|c|c|c|c|c|}
\hline Reference & Patient & $\begin{array}{c}\text { Diagnosis } \\
\text { (past history) }\end{array}$ & $\begin{array}{l}\text { Sampling } \\
\text { location }\end{array}$ & Co-infections & $\begin{array}{l}\text { Antibiotic } \\
\text { treatment }\end{array}$ & Antibiotic susceptibilities & $\begin{array}{l}\text { Identification } \\
\text { method }\end{array}$ \\
\hline Present study & $51 / \mathrm{F}$ & $\begin{array}{l}\text { Chronic ear disease (s/p } \\
\text { canal wall-down } \\
\text { mastoidectomy, both, } \\
\text { approximately } 2 \text { yr ago) }\end{array}$ & $\begin{array}{l}\text { Left external } \\
\text { auditory } \\
\text { meatus }\end{array}$ & Diphtheroid & $\begin{array}{l}\text { Oral cefpodoxime } \\
\text { and cefcapene }\end{array}$ & $\begin{array}{l}\text { Susceptible to amikacin, aztreonam, } \\
\text { cefepime, cefotaxime, ceftazidime, } \\
\text { gentamicin, imipenem, levofloxacin, } \\
\text { meropenem, piperacillin-tazobactam, } \\
\text { piperacillin, ticarcillin-clavulanate, } \\
\text { tobramycin, and trimethoprim- } \\
\text { sulfamethoxazole and resistant to } \\
\text { ciprofloxacin }\end{array}$ & $\begin{array}{l}\text { MALDI-TOF MS, } \\
\text { confirmed by } \\
\text { 16S rRNA gene } \\
\text { sequencing }\end{array}$ \\
\hline $\begin{array}{c}\text { Almuzara et al } \\
\text { (2012) [4] }\end{array}$ & $16 / \mathrm{M}$ & $\begin{array}{l}\text { Complicated } \\
\text { cholesteatomatous } \\
\text { chronic otitis media with } \\
\text { left peripheral facial } \\
\text { palsy grade IV (Left otitis } \\
\text { media at age } 12 \text { and } \\
\text { retroauricular abscess) }\end{array}$ & $\begin{array}{l}\text { Bezold's } \\
\text { abscess }\end{array}$ & None & $\begin{array}{l}\text { Intravascular } \\
\text { ampicillin- } \\
\text { sulbactam and } \\
\text { ceftriaxone/oral } \\
\text { ciprofloxacin and } \\
\text { amoxicillin- } \\
\text { clavulanic acid }\end{array}$ & $\begin{array}{l}\text { Susceptible to amoxicillin, ceftriaxone, } \\
\text { ceftazidime, cefepime, trimethoprim- } \\
\text { sulfamethoxazole, ciprofloxacin, and } \\
\text { levofloxacin }\end{array}$ & $\begin{array}{l}\text { 16S rRNA gene } \\
\text { sequencing }\end{array}$ \\
\hline $\begin{array}{l}\text { Pence et al } \\
\text { (2013) [6] }\end{array}$ & $55 / \mathrm{M}$ & $\begin{array}{l}\text { Chronic ear disease (s/p } \\
\text { canal wall-down } \\
\text { mastoidectomy, both, } \\
\text { approximately } 40 \text { yr ago) }\end{array}$ & $\begin{array}{l}\text { Left mastoid } \\
\text { cavity }\end{array}$ & $\begin{array}{l}\text { Corynebacterium } \\
\text { amycolatum }\end{array}$ & $\begin{array}{l}\text { Trimethoprim- } \\
\text { sulfamethoxazole }\end{array}$ & $\begin{array}{l}\text { Susceptible to cefepime, piperacillin- } \\
\text { tazobactam, Trimethoprim- } \\
\text { sulfamethoxazole, and gentamicin and } \\
\text { resistant to ciprofloxacin }\end{array}$ & $\begin{array}{l}\text { MALDI-TOF MS, } \\
\text { confirmed by } \\
\text { 16S rRNA gene } \\
\text { sequencing }\end{array}$ \\
\hline $\begin{array}{l}\text { Mwalutende } \\
\text { et al (2014) } \\
\text { [5] }\end{array}$ & $53 / \mathrm{M}$ & $\begin{array}{l}\text { Chronic suppurative otitis } \\
\text { media }\end{array}$ & $\begin{array}{l}\text { Left external } \\
\text { auditory } \\
\text { meatus }\end{array}$ & Proteus mirabilis & $\begin{array}{l}\text { Ciprofloxacin ear } \\
\text { drops }\end{array}$ & $\begin{array}{l}\text { Susceptible to piperacillin, cefotaxime, } \\
\text { ceftazidime, gentamicin, imipenem, } \\
\text { meropenem, and moxifloxacin and } \\
\text { partially susceptible to ciprofloxacin }\end{array}$ & MALDI-TOF MS \\
\hline $\begin{array}{l}\text { Mwalutende } \\
\text { et al (2014) } \\
\text { [5] }\end{array}$ & 33/M & $\begin{array}{l}\text { Chronic suppurative otitis } \\
\text { media }\end{array}$ & $\begin{array}{l}\text { Right } \\
\text { external } \\
\text { auditory } \\
\text { meatus }\end{array}$ & $\begin{array}{l}\text { Staphylococcus } \\
\text { aureus, } \\
\text { Escherichia } \\
\text { coli }\end{array}$ & $\begin{array}{l}\text { Ciprofloxacin ear } \\
\text { drops }\end{array}$ & $\begin{array}{l}\text { Susceptible to piperacillin, cefotaxime, } \\
\text { ceftazidime, gentamicin, imipenem, } \\
\text { meropenem, and moxifloxacin and } \\
\text { partially susceptible to ciprofloxacin }\end{array}$ & MALDI-TOF MS \\
\hline $\begin{array}{l}\text { Uysal et al } \\
\text { (2015) [7] }\end{array}$ & $25 / \mathrm{M}$ & $\begin{array}{l}\text { Chronic suppurative otitis } \\
\text { media }\end{array}$ & $\begin{array}{l}\text { Right } \\
\text { external } \\
\text { auditory } \\
\text { meatus }\end{array}$ & $\begin{array}{c}\text { Pseudomonas } \\
\text { aeruginosa }\end{array}$ & $\begin{array}{l}\text { Intravascular } \\
\text { imipenem }\end{array}$ & $\begin{array}{l}\text { Susceptible to amikacin, cefepime, } \\
\text { ceftazidime, colistin, imipenem, } \\
\text { meropenem, and piperacillin- } \\
\text { tazobactam, intermediate to levofloxacin, } \\
\text { and ticarcillin-clavulanate and resistant } \\
\text { to aztreonam, ciprofloxacin, and } \\
\text { trimethoprim-sulfamethoxazole }\end{array}$ & $\begin{array}{l}\text { MALDI-TOF MS, } \\
\text { confirmed by } \\
\text { 16S rRNA gene } \\
\text { sequencing }\end{array}$ \\
\hline
\end{tabular}

Abbreviations: F, female; M, male; MALDI-TOF MS, matrix-assisted laser desorption ionization-time of flight mass spectrometry. 
Enterobacteriaceae [9]. The isolate was susceptible to amikacin (MIC $\leq 16 \mu \mathrm{g} / \mathrm{mL})$, aztreonam $(\leq 4 \mu \mathrm{g} / \mathrm{mL})$, cefepime ( $\leq 2 \mu \mathrm{g} /$ $\mathrm{mL}$ ), cefotaxime $(\leq 1 \mu \mathrm{g} / \mathrm{mL})$, ceftazidime $(\leq 1 \mu \mathrm{g} / \mathrm{mL})$, gentami$\operatorname{cin}(\leq 4 \mu \mathrm{g} / \mathrm{mL})$, imipenem $(\leq 1 \mu \mathrm{g} / \mathrm{mL})$, levofloxacin $(\leq 2 \mu \mathrm{g} /$ $\mathrm{mL}$ ), meropenem $(\leq 1 \mu \mathrm{g} / \mathrm{mL})$, piperacillin-tazobactam $(\leq 16$ $\mu \mathrm{g} / \mathrm{mL}$ ), piperacillin $(\leq 16 \mu \mathrm{g} / \mathrm{mL})$, ticarcillin-clavulanate $(\leq 16$ $\mu \mathrm{g} / \mathrm{mL}$ ), tobramycin $(\leq 4 \mu \mathrm{g} / \mathrm{mL})$, and trimethoprim-sulfamethoxazole $(\leq 2 / 38 \mu \mathrm{g} / \mathrm{mL})$ and was resistant to ciprofloxacin ( $>2 \mu \mathrm{g} / \mathrm{mL}$ ).

A week after the initial visit, the antibiotic administered was changed to cefcapene and continued for six days; four weeks later, the patient's ear discharge symptoms were resolved with no other complications.

K. gyiorum has been isolated from patients with various diseases [1]. A total of 10 cases of patients infected with K. gyiorum have been recorded, five of which were associated with COM; the features of these cases are shown in Table 1 [4-7]. Four of the five patients showed co-infection, which is a common feature of COM [10]; likewise, a diphtheroid was isolated from our patient with $K$. gyiorum who developed an infection at the site of operation approximately 25 years after undergoing surgery for COM.

It is difficult to distinguish $\mathrm{K}$. gyiorum from other microorganisms using biochemical methods as it has characteristics similar to those of other microorganisms [4]. Additionally, K. gyiorum is not accurately identified by automated bacterial identification methods such as MicroScan Walkaway, Vitek 2, API 20 NE (bioMérieux), and the RapID NF plus assay (Thermo Fisher Scientific, Lenexa, KS, USA) [4-8]. MALDI-TOF MS, which has recently been widely used in clinical laboratories, identified K. gyiorum, both in our case and in previously reported cases [5-8]. Therefore, MALDI-TOF MS should be used when bacterial species identification is difficult using conventional methods. In addition, the clinician and laboratory should consider the possibility of $K$. gyiorum infection and the use of MALDI-TOF MS when inaccurate identification of pathogen is suspected in patients with COM.

\section{Authors' Disclosures of Potential Conflicts of Interest}

No potential conflicts of interest relevant to this article are reported.

\section{REFERENCES}

1. Baran I, Düzqün AP, Mumcuo ğlu I, Aksu N. Chronic lower extremity wound infection due to Kerstersia gyiorum in a patient with Buerger's disease: a case report. BMC Infect Dis 2017;17:608.

2. Coenye T, Vancanneyt M, Cnockaert MC, Falsen E, Swings J, Vandamme P. Kerstersia gyiorum gen. nov., sp. nov., a novel Alcaligenes faecalis-like organism isolated from human clinical samples, and reclassification of Alcaligenes denitrificans Ruger and Tan 1983 as Achromobacter denitrificans comb. nov. Int J Syst Evol Microbiol 2003;53:182531.

3. Austin B. The family Alcaligenaceae. In: Rosenberg E, DeLong EF, et al., eds. The prokaryotes: alphaproteobacteria and betaproteobacteria. Berlin, Heidelberg: Springer Berlin Heidelberg, 2014:753-54.

4. Almuzara MN, Barberis CM, Traglia GM, Ordoñez AM, Famiglietti AM, Ramirez MS, et al. Isolation of Kerstersia gyiorum from a patient with cholesteatomatous chronic otitis media. J Clin Microbiol 2012;50:380911.

5. Mwalutende A, Mshana SE, Mirambo MM, Mushi MF, Chalya PL, Giliyoma JM, et al. Two cases of chronic suppurative otitis media caused by Kerstersia gyiorum in Tanzania: is it an underappreciated pathogen in chronic otitis media? Int J Infect Dis 2014;29:251-3.

6. Pence MA, Sharon J, McElvania Tekippe E, Pakalniskis BL, Ford BA, Burnham CA. Two cases of Kerstersia gyiorum isolated from sites of chronic infection. J Clin Microbiol 2013;51:2001-4.

7. Uysal EB, Çelik C, Tuzcu N, Can F, Doğan M, Ertürk R, et al. A case of chronic suppurative otitis media caused by Kerstersia gyiorum. APMIS 2015; 123:986-9.

8. Ogawa Y, Lee ST, Kasahara K, Koizumi A, Chihara Y, Nakano R, et al. A first case of isolation of Kerstersia gyiorum from urinary tract. J Infect Chemother 2016;22:265-7.

9. CLSI. Performance standards for antimicrobial susceptibility testing. 27th ed, CLSI supplement M100S. Wayne, PA: Clinical and Laboratory Standards Institute; 2017.

10. Vartiainen E and Vartiainen J. Effect of aerobic bacteriology on the clinical presentation and treatment results of chronic suppurative otitis media. J Laryngol Otol 1996;110:315-8. 